\title{
The Prognostic Value of Sex-Determining Region Y-Box 2 and CD8+ Tumor-Infiltrating Lymphocytes in Limited-Stage Small-Cell Lung Cancer
}

\author{
Jinsoo Lee ${ }^{a, b}$ Yoon Yang Jung ${ }^{c}$ Jung Hoon Lee ${ }^{d}$ Mineui Hong ${ }^{e}$ \\ Hye-Won Hwang $^{f}$ Soon Auck Hong ${ }^{f}$ Sook-Hee Hong ${ }^{a}$
}

\begin{abstract}
aDivision of Medical Oncology, Department of Internal Medicine, The Catholic University of Korea, Seoul, Republic of Korea; bivision of Medical Oncology, Department of Internal Medicine, Good Morning Hospital, Pyeontaek-si, Republic of Korea; 'Department of Pathology, Myongji Hospital, Hanyang University College of Medicine, Goyang, Republic of

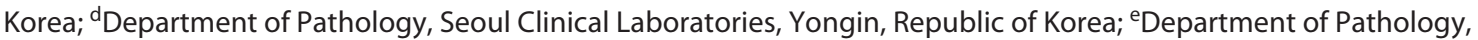
Kangnam Sacred Heart Hospital, Hallym University College of Medicine, Seoul, South Korea; fDepartment of Pathology, College of Medicine, Chung-Ang University, Seoul, South Korea
\end{abstract}

\section{Keywords}

Sex-determining region Y-box $2 \cdot C D 8+$ tumor-infiltrating lymphocyte $\cdot$ Small-cell lung cancer

\begin{abstract}
Background: Sex-determining region Y-box 2 (SOX2) is a transcriptional factor that drives embryonic stem cells to neuroendocrine cells in lung development and is highly expressed in small-cell lung cancer (SCLC). However, the prognostic role of SOX2 and its relationship with tumor-infiltrating lymphocytes (TILs) has not been determined in SCLC. Herein, we assessed the expression of SOX2 and CD8+ TILs to obtain insights into the prognostic role of SOX 2 and CD8+ TILs in limited-stage (LS)-SCLC. Methods: A total of 75 patients with LS-SCLC was enrolled. The SOX2 expression and CD8+ TILs were evaluated by immunohistochemistry. $\boldsymbol{R e}$ sults: High SOX2 and CD8+ TIL levels were identified in 52 (69.3\%) and 40 (53.3\%) patients, respectively. High SOX2 expression was correlated with increased density of CD8+ TILs $(p=0.041)$. Unlike SOX2, high CD8+ TIL numbers were associated with significantly longer progression-free survival (PFS; 13.9 vs. 8.0 months, $p=0.014$ ). Patients with both high SOX2 expression and CD8+ TIL numbers $(n=29,38.7 \%)$ had
\end{abstract}

karger@karger.com www.karger.com/ocl

Karger $\stackrel{\text { ' }}{5}$

GOPEN ACCESS
(C) 2021 The Author(s)

Published by S. Karger AG, Basel

This is an Open Access article licensed under the Creative Commons Attribution-NonCommercial-4.0 International License (CC BY-NC) (http://www.karger.com/Services/OpenAccessLicense), applicable to the online version of the article only. Usage and distribution for commercial purposes requires written permission. significantly longer PFS and overall survival (OS) compared to those from the other groups (median PFS 19.3 vs. 8.4 months; $p=0.002$ and median OS 35.7 vs. 17.4 months; $p=$ 0.004 , respectively). Multivariate Cox regression analysis showed that the combination of high SOX2 expression and CD8+ TIL levels was an independent good prognostic factor for OS (HR $=0.471,95 \% \mathrm{Cl}, 0.250-0.887, p=0.02)$ and PFS $(\mathrm{HR}=0.447,95 \% \mathrm{Cl}, 0.250-0.801, p=0.007)$ in SCLC. Conclusions: Evaluation of the combination of SOX 2 and CD8+ TIL levels may be of a prognostic value in LS-SCLC.

\section{(c) 2021 The Author(s)}

Published by S. Karger AG, Basel

\section{Introduction}

Small-cell lung cancer (SCLC) is a malignant tumor originating from pulmonary neuroendocrine cells that is characterized by fast growth and early widespread metastasis; it has a dismal prognosis [1]. Consequently, curative surgical resection, which is the gold standard treatment modality for most other cancers, is not feasible in most cases of SCLCs. SCLC treatment is relatively standard and consists of chemotherapy, including treatment with etoposide and platinum, with/without radiation and has
Correspondence to:

Soon Auck Hong, hsu108@ cau.ac.kr

Sook-Hee Hong, ssuki76@catholic.ac.kr 
been the same since the early 1990s. The overall response rate for these treatments is approximately $90 \%$, but more than half of the patients relapse in 2 years, resulting in a median overall survival (OS) of only 20 months [2, 3]. Based on staging, SCLC is classified into 2 stages: limited and extensive stage. Limited-stage SCLC (LS-SCLC) is defined as a tumor that is confined to one hemithorax, usually with one single radiation port and no pleural effusion, while in extensive-stage SCLC (ES-SCLC) the disease extends beyond one radiation field and is generally accompanied by metastasis and pleural effusion [1]. ESSCLC generally has a poor prognosis with a median OS of $<10$ months and a 5-year survival rate of $<5 \%$ [4], whereas LS-SCLC has a 5 -year survival rate of $20-40 \%$ [5].

Recently, etoposide and platinum with anti-programed death (PD) ligand 1 immunotherapy (atezolizumab or duvalumab) have been approved as a new standard therapy in ES-SCLC $[6,7]$. However, current standard treatment in LS-SCLC is still concurrent etoposide and platinum chemoradiotherapy for decades. Combination immunotherapy such as anti- $\mathrm{PD}(\mathrm{L}) 1$ and/or anticytotoxic T-lymphocyte-associated protein 4 has been evaluated in LS-SCLC with chemoradiotherapy or as consolidation therapy, however the role of immunotherapy in LS-SCLC has not yet defined [8].

The core concept of immunotherapy is based on the tumoricidal effect of CD8+ T-cells [9]. These findings are supported by numerous studies, showing that CD8+ tumor-infiltrating lymphocytes (CD8+ TILs) are associated with good prognosis $[10,11]$. Although a complex and delicate mechanism is suggested to regulate effective CD8+ TIL response in solid tumors, tumor antigens have been suggested as regulators of antitumor immunity associated with CD8+ TILs in solid tumors [12].

Sex-determining region Y-box 2 (SOX2) is a transcriptional factor that regulates neuronal differentiation of pluripotent stem cells and differentiation of embryonic stem cells to neuroendocrine cells during lung development [13]. In SCLC, SOX2 is frequently amplified and present in approximately $25 \%$ of SCLC tumor samples [14]. However, the prognostic role of SOX2 in SCLC has not been studied extensively. Apart from the oncogenic role of SOX2 in SCLC, immunoreactive antibodies for SOX2 have been reported in the serum of patients with solid tumors, including SCLC [15-17]. Recent studies have also shown that the SOX2 tumor antigen is a target for cytotoxic T-cells [18]. However, the role of the combination of SOX2 expression and CD8+ TIL numbers in SCLC has not been studied previously.
Table 1. Baseline characteristics of LS-SCLC

\begin{tabular}{ll}
\hline Varibles & All patients $(n=75)$ \\
\hline $\begin{array}{l}\text { Median age (range), years } \\
\text { Sex }\end{array}$ & $70(44-85)$ \\
$\quad$ Male & $64(85.3 \%)$ \\
Female & $11(14.7 \%)$ \\
Smoking status & $64(85.3 \%)$ \\
$\quad$ Current or former smoker & $5(6.7 \%)$ \\
$\quad$ Never smoker & $6(8 \%)$ \\
Unknown & \\
ECOG PS & $63(84 \%)$ \\
$\quad 0,1$ & $12(16 \%)$ \\
2 & \\
Histology type & $62(82.7 \%)$ \\
$\quad$ SCLC & $13(17.3 \%)$ \\
SCLC with NSCLC combined & $30(40 \%)$ \\
Serum LDH level & $45(60 \%)$ \\
$\quad<$ UNL (450 U/L) & \\
$\quad$ UNL (450 U/L) & $46(61.3 \%)$ \\
Treatment modality & $18(24 \%)$ \\
CCRT or sequential CT and RT & $11(14.7 \%)$ \\
Surgery and CT \pm RT & $45(24-55)$ \\
CT or RT alone & $31(41.3 \%)$ \\
Median RT dose (range), Gy & $44(58.7 \%)$ \\
PCI & $38(50.7 \%)$ \\
Yes & $37(49.3 \%)$ \\
No & \\
Yubsequent CT & \\
No & \\
\hline &
\end{tabular}

ECOG PS, Eastern Cooperative Oncology Group Performance status; LDH, lactate dehydrogenase; PCI, prophylactic cranial irradiation; CT, chemotherapy; $\mathrm{RT}$, radiation therapy; CCRT, concurrent chemoradiation therapy; SCLC, small-cell lung cancer; LSSCLC, limited-stage small-cell lung cancer; NSCLC, non-small cell lung cancer.

In this study, we assessed the combined effect of SOX2 expression in tumor cells and CD8+ TIL numbers in 75 patients with LS-SCLC. Further, we investigated the correlation between SOX2/CD8+ TIL expression, clinicopathologic factors, and survival of patients with LS-SCLC.

\section{Materials and Methods}

\section{Study Population}

We retrospectively reviewed data from histologically confirmed LS-SCLC patients treated between January 1, 2010, and August 31, 2018, at the Seoul St. Mary's Hospital, Korea. Among the total of 245 patients treated for LS-SCLC, 41 patients were referred to our hospital after diagnosed with SCLC at outside institutions, and 21 patients refused cancer therapy. Among the 183 patients who received treatment of LS-SCLC, 25 patients were di- 
Table 2. Correlation between SOX2, CD8+TIL, and clinicopathologic findings of LS-SCLC

\begin{tabular}{|c|c|c|c|c|c|c|c|}
\hline Variable & $\begin{array}{l}\text { Total } \\
(n=75)(\%)\end{array}$ & \multicolumn{3}{|l|}{ SOX2 } & \multicolumn{3}{|l|}{ CD8+ TIL } \\
\hline \multicolumn{8}{|l|}{ Age } \\
\hline$\geq 65$ years & $51(68)$ & $34(65.3)$ & $17(73.9)$ & \multirow{2}{*}{0.465} & $30(75.0)$ & $21(60.0)$ & \multirow{2}{*}{0.165} \\
\hline$<65$ years & $24(32)$ & $18(34.6)$ & $6(26.1)$ & & $10(25.0)$ & $14(40.0)$ & \\
\hline Female & $11(24.7)$ & $8(15.4)$ & $3(13.0)$ & 0.792 & $7(17.5)$ & $4(11.4)$ & 0.458 \\
\hline \multicolumn{8}{|l|}{ Smoking status } \\
\hline Current or former smoker & $64(85.3)$ & $44(84.6)$ & $20(87.0)$ & \multirow{3}{*}{0.681} & $35(87.5)$ & $29(82.9)$ & \multirow{3}{*}{0.577} \\
\hline Never smoker & $5(6.7)$ & $3(5.8)$ & $2(8.7)$ & & $3(7.5)$ & $2(5.7)$ & \\
\hline Unknown & $6(8)$ & $5(9.6)$ & $1(4.3)$ & & $2(5)$ & $4(11.4)$ & \\
\hline \multicolumn{8}{|l|}{ ECOG PS } \\
\hline$\geq \mathrm{UNL}(450 \mathrm{U} / \mathrm{L})$ & $45(60)$ & $29(55.8)$ & $16(69.6)$ & 0.261 & $16(40.0)$ & $14(40.0)$ & 1 \\
\hline \multicolumn{8}{|l|}{ Treatment modality } \\
\hline CCRT or surgery and CT $\pm \mathrm{RT}$ & $59(78.7)$ & $39(75.0)$ & $20(87.0)$ & \multirow{2}{*}{0.390} & $32(80.0)$ & $27(77.1)$ & \multirow{2}{*}{0.785} \\
\hline Sequential CT and RT or CT/RT alone & $16(21.3)$ & $13(25.0)$ & $3(13.0)$ & & $8(20.0)$ & $8(22.9)$ & \\
\hline \multicolumn{8}{|c|}{ PCI } \\
\hline Yes & $31(41.3)$ & $21(40.4)$ & $10(43.5)$ & \multirow{2}{*}{0.802} & $15(37.5)$ & $16(45.7)$ & \multirow{2}{*}{0.471} \\
\hline No & $44(58.7)$ & $31(59.6)$ & $13(56.5)$ & & $25(62.5)$ & $19(54.3)$ & \\
\hline \multicolumn{8}{|l|}{ Subsequent CT } \\
\hline Yes & $38(50.7)$ & $25(48.1)$ & $13(56.5)$ & \multirow{2}{*}{0.5} & $17(42.5)$ & $21(60.0)$ & \multirow{2}{*}{0.13} \\
\hline No & $37(49.3)$ & $27(51.9)$ & $10(43.5)$ & & $23(57.5)$ & $14(40.0)$ & \\
\hline
\end{tabular}

ECOG PS, Eastern Cooperative Oncology Group Performance status; LDH, lactate dehydrogenase; PCI, prophylactic cranial irradiation; CT, chemotherapy; RT, radiation therapy; CCRT, concurrent chemoradiation therapy; LS-SCLC, limited-stage small-cell lung cancer; TIL, tumor-infiltrating lymphocyte; SOX2, sex-determining region Y-box 2.

agnosed with LS-SCLC by cytologic examination of endoscopic bronchial ultrasonography-fine needle aspiration or bronchoscopic evaluation. Only 75 of 158 patients were finally enrolled in this study due to paraffin blocks' availability for immunohistochemistry. Clinical data, including age, sex, Eastern cooperative oncology group performance status, smoking status, the serum LDH level, treatment modality, survival time, and survival status were retrieved from their medical records. This study was approved by the Institutional Review Board of the Seoul St. Mary's Hospital, and informed consent was waived due to the retrospective nature of the study.

\section{Immunohistochemistry}

The numbers of CD8+ TILs and expression levels of SOX2 were evaluated by immunohistochemistry. Immunohistochemistry analysis was performed using 4 - $\mu$ m-thick paraffin-embedded tissue sections, using the following primary antibodies: anti-SOX2 (1:50, SP76, Abcam, Cambridge, UK) and anti-CD8 (1:100, C8/144B, Dako, Cambridge, UK), and Ventana BenchMark XT stainer (Ventana, Tucson, AZ, USA), according to the manufacturer's instructions. CD8+ TIL numbers and SOX2 expression were evaluated independently by 2 board-certified pathologists (S.A.H. and H.W.H.) in a blinded manner. The SOX2 expression was graded as the staining intensity and proportion of stained cells. Staining intensity was scored as follows: weak $=1$; moderate $=2$; strong = 3; and the proportion of stained cells was evaluated as the percentage of stained cells. Various scores, derived from multiplying the staining intensity by the proportion of stained tumor cells, were tested to determine a cut value for the high and low expression. High SOX2 expression was defined as a score above 100 that was best predicted the patient's survival, using the Kaplan-Meier method and log-rank test. The evaluation of CD8+ TIL was performed according to a previous study [19]. The number of CD8+ TILs was counted under high magnification $(\times 400)$ in 5 fields in the intratumoral area, and the mean number of CD8+ TILs was calculated. The cutoff value for high CD8+ TIL numbers was above the median values of the total cases.

\section{Statistical Analysis}

SOX2 and CD8+ TIL levels were compared with baseline factors using the $\chi^{2}$ test. OS was defined as the duration between the date of the start of treatment for LS-SCLC and that of death from 


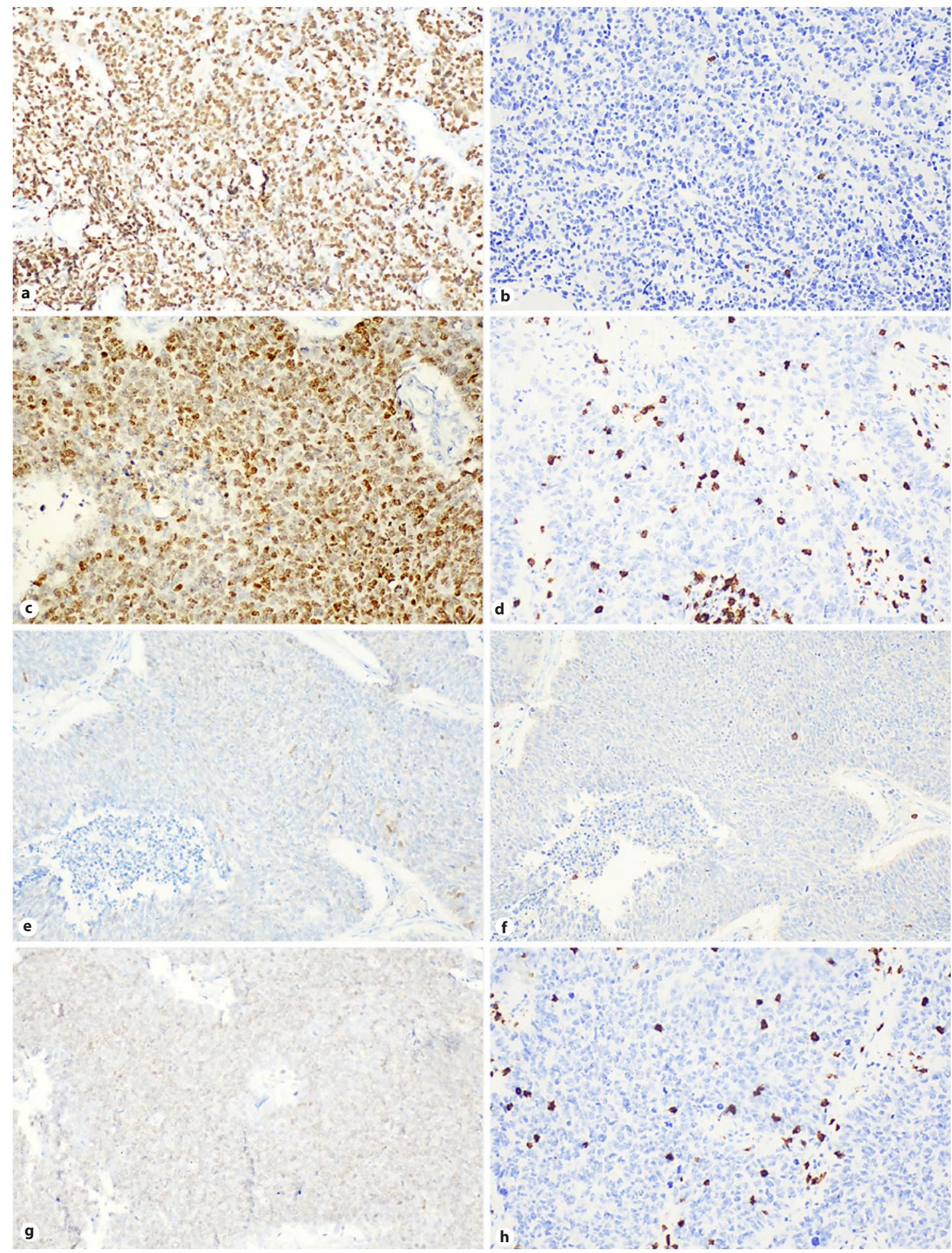

Fig. 1. Representative images of the immunohistochemical staining of SOX2 in tumor cells and CD8+ TILs. High SOX2 expression in tumor cells (a) and low levels of CD8+ TILs (b). High SOX2 expression in tumor cells (c) and high levels of CD8+ TILs (d). Low SOX2 expression in tumor cells (e) and low levels of CD8+ TILs (f). Low SOX2 expression of tumor cells (g) and high numbers of CD8+ TILs (h). TILs, tumor-infiltrating lymphocytes; SOX2, sex-determining region Y-box 2. 
any cause, while the progression-free survival (PFS) was defined as the duration between the date of start of treatment and that of the detection of disease progression on radiologic exam. The OS and PFS of patients based on the expression of SOX2 and CD8+ TILs were estimated using the Kaplan-Meier method, and the log-rank test was used to compare survival curves. Univariate and multivariate analyses using Cox proportional hazard regression models were performed to examine prognostic factors for PFS and OS. $p<0.05$ was considered statistically significant. All statistical analyses were performed using SPSS version 24.00 (IBM Corp, Armonk, NY, USA) and R statistical programming version 3.4.1 (http://www.r-project.org).

\section{Results}

\section{Patient Characteristics}

The baseline characteristics of patients are summarized in Table 1 . The median age was 70 years (range, $44-85$ years), and $85.3 \%$ of patients were male. A total of $46(61.3 \%)$ patients were treated with etoposide/platinum concurrent chemoradiation or chemotherapy with sequential radiation therapy, while $18(24 \%)$ patients received surgical resection with chemotherapy with/without radiation therapy. The median OS of the entire patient population was 19.3 months; the median PFS was 10.3 months.

SOX2 Expression and CD8+ TIL Numbers in LS-SCLC

Table 2 shows the patient characteristics according to the SOX2 expression and CD8+ TIL levels detected using immunohistochemistry. High SOX2 expression was observed in 52 patients $(69.3 \%)$. The median number of CD8+ TILs was 13 (range, 1-115). SOX2 expression and CD8+ TIL levels did not differ with regard to any clinical variable. Based on the cut-off value for high and low CD8+ TIL numbers, a high CD8+ TIL level was identified in $40(53.3 \%)$ patients. Based on the SOX2 expression and CD8+ TIL numbers, the patients were classified into the following 4 groups: high SOX2/low CD8+ TIL, high SOX2/high CD8+ TIL, low SOX2/low CD8+ TIL, and low SOX2/high CD8+ TIL (Fig. 1a-h). The number of CD8+ TILs in the high SOX2 group was significantly higher than that in the low SOX2 group ( $p=0.041)$ (Fig. 2). High SOX2/high CD8+ TIL levels were found in 29 patients (38.7\%) and were not significantly related to any clinicopathologic features (Table 3).

\section{Effects of SOX2 Expression and CD8+ TIL Numbers on Survival}

The OS and PFS of the high SOX2 group were longer than those of the low SOX2 group, but the differences

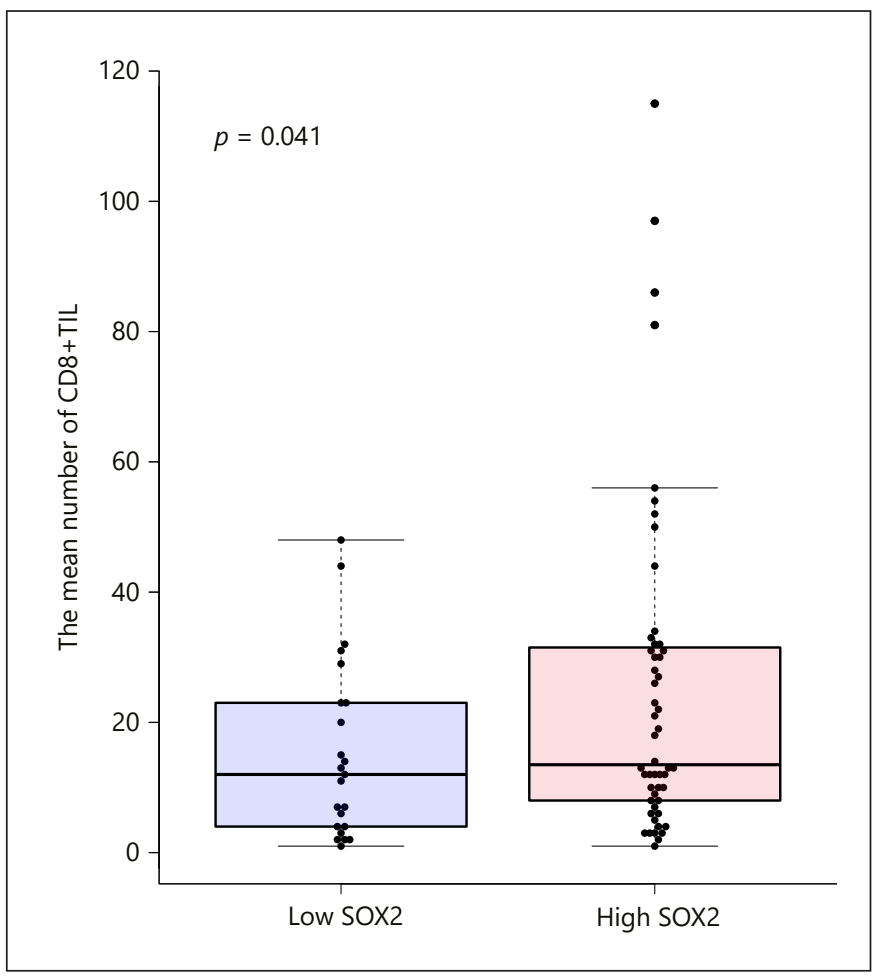

Fig. 2. Differences in the numbers of CD8+ TILs. The mean number of CD8+ TILs in the group with high SOX2 expression in the tumor is significantly higher than those in the group with low SOX2 expression $(p=0.041)$. TILs, tumor-infiltrating lymphocytes; SOX2, sex-determining region Y-box 2.

were not statistically significant (median OS 21.7 vs. 17.1 months, $p=0.118$; median PFS 12.7 vs. 9.0 months, $p=$ 0.11) (Fig. 3a, b). Patients with high CD8+ TIL numbers had a longer OS and PFS than the low CD8+ TIL group. Although the difference in OS was not significant $(p=$ 0.058) (Fig. 3c), the difference in PFS was statistically significant (13.9 vs. 8.0 months, $p=0.014$ ) (Fig. $3 \mathrm{~d}$ ).

On combined analysis of SOX2 expression and CD8+ TIL numbers, patients with high SOX2/high CD8+ TIL demonstrated significantly longer OS $(p=0.004)$ (Fig. 4a) and PFS ( $p=0.001$ ) (Fig. 4b). The differences in survival among the 4 groups (high SOX2/high CD8+ TIL, high SOX2/low CD8+ TIL, low SOX2/high CD8+ TIL, and low SOX2/low CD8+ TIL), were compared and OS $(p=0.032)$ (Fig. 3c) and PFS ( $p=0.024$ ) (Fig. 3d) were found to be significantly different among the 4 groups.

\section{Univariate and Multivariate Analyses}

Univariate and multivariate analyses of the baseline characteristics of OS and PFS were performed (Table 4). In the univariate analysis, Eastern cooperative oncology group 
Table 3. Association between combined high SOX2/CD8+ TIL and clinicopathologic findings of LS-SCLC

\begin{tabular}{|c|c|c|c|}
\hline \multirow[t]{2}{*}{ Variable } & \multicolumn{3}{|c|}{ Combined high SOX2/CD8+ TIIL } \\
\hline & yes $(n=29 ; 38.7 \%)$ & no $(n=46 ; 61.3 \%)$ & $p$ value \\
\hline \multicolumn{4}{|l|}{ Age, years } \\
\hline$\geq 65$ & $23(79.3 \%)$ & $28(60.9 \%)$ & \multirow{2}{*}{0.158} \\
\hline$<65$ & $6(20.7 \%)$ & $18(39.1 \%)$ & \\
\hline \multicolumn{4}{|l|}{ Sex } \\
\hline Male & $24(82.8 \%)$ & $40(87.0 \%)$ & \multirow{2}{*}{0.869} \\
\hline Female & $5(17.2)$ & $6(13.0 \%)$ & \\
\hline \multicolumn{4}{|l|}{ Smoking status } \\
\hline Current or former smoker & $25(86.2 \%)$ & $39(84.8 \%)$ & \multirow{3}{*}{0.961} \\
\hline Never smoker & $2(6.9 \%)$ & $3(6.5 \%)$ & \\
\hline Unknown & $2(6.9 \%)$ & $4(8.7 \%)$ & \\
\hline \multicolumn{4}{|l|}{ ECOG PS } \\
\hline 0,1 & $26(89.7 \%)$ & $37(80.4 \%)$ & \multirow[b]{2}{*}{0.461} \\
\hline 2 & $3(10.3 \%)$ & $9(19.6 \%)$ & \\
\hline \multicolumn{4}{|l|}{ Serum LDH level } \\
\hline$<\mathrm{UNL}(450 \mathrm{U} / \mathrm{L})$ & $12(41.4 \%)$ & $18(39.1 \%)$ & \multirow[b]{2}{*}{1} \\
\hline$\geq \mathrm{UNL}(450 \mathrm{U} / \mathrm{L})$ & $17(58.6 \%)$ & $28(60.9 \%)$ & \\
\hline \multicolumn{4}{|l|}{ Treatment modality } \\
\hline CCRT or surgery and CT \pm RT & $23(79.3 \%)$ & $36(78.3 \%)$ & \multirow[b]{2}{*}{1} \\
\hline Sequential CT and RT or CT/RT alone & $6(20.7 \%)$ & $10(21.7 \%)$ & \\
\hline \multicolumn{4}{|l|}{ PCI } \\
\hline Yes & $9(31.0 \%)$ & $22(47.8 \%)$ & \multirow{2}{*}{0.231} \\
\hline No & $20(69.0 \%)$ & $24(52.2 \%)$ & \\
\hline \multicolumn{4}{|l|}{ Subsequent CT } \\
\hline Yes & $11(37.9 \%)$ & $27(58.7 \%)$ & \multirow{2}{*}{0.13} \\
\hline No & $18(62.1 \%)$ & $19(41.3 \%)$ & \\
\hline
\end{tabular}

ECOG PS, Eastern Cooperative Oncology Group Performance status; LDH, lactate dehydrogenase; PCI, prophylactic cranial irradiation; CT, chemotherapy; RT, radiation therapy; CCRT, concurrent chemoradiation therapy; LS-SCLC, limited-stage small-cell lung cancer; TIL, tumor-infiltrating lymphocyte; SOX2, sex-determining region Y-box 2.

performance status, the serum $\mathrm{LDH}$ level, and the combination of SOX2 expression and CD8+ TIL numbers were statistically significant factors for OS and PFS. In the multivariate analysis of OS, high expression levels of SOX2 and CD8+ TIL numbers were a good prognostic factor (HR $0.471,95 \%$ CI $0.250-0.887, p=0.02$ ). In the multivariate analysis of PFS, the combination of high SOX2 expression levels and CD8+ TIL numbers were independent favorable variables (HR 0.447, 95\% CI 0.250-0.801, $p=0.007$ ).

\section{Discussion}

SCLC is a highly aggressive neuroendocrine tumor, characterized by rapid progression and distant metastasis; LS-SCLC is a relatively rare and curable disease [20]. Prognostic factors for LS-SCLC have not been extensively studied until recently. In our study, we analyzed the expression of SOX2 and CD8+ TILs in LS-SCLC. Although the SOX2 expression was not associated with OS and PFS, patients with concomitantly high SOX2 and CD8+ TIL levels demonstrated longer OS and PFS. Notably, high SOX2 and CD8+ TIL levels were independent prognostic factors for PFS and OS.

CD8+ TILs are the most important effector immune cells that eradicate tumor cells in various solid tumors and are associated with favorable patient prognosis. In a previous study, increased T-cell density and CD3 and CD8 immunostaining were related to favorable prognosis in ES-SCLC [21]. High CD8+ TIL number was also recognized as a good prognostic factor, with the combined analysis of either galectin-9 or PD-L1 expression in SCLC $[22,23]$. The above result of the prognostic role for CD8+ TILs has been obtained from ES-SCLC cases with/without LS-SCLC. However, the prognostic role of CD8+ TILs in LS-SCLC has not been well defined. Thus, the finding 


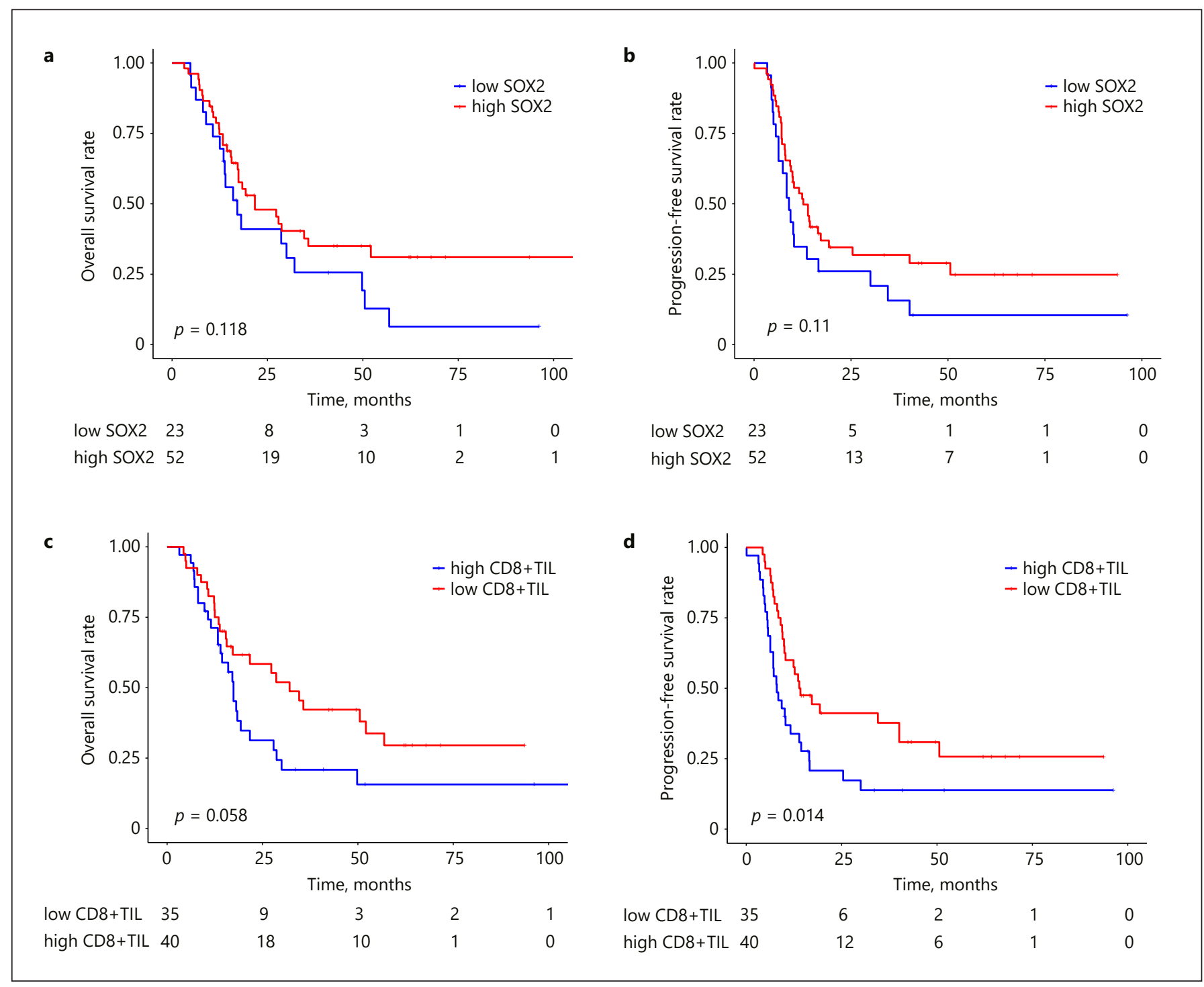

Fig. 3. Kaplan-Meier curves and log-rank analysis. OS (a) and PFS (b) curves according to SOX 2 expression. OS (c) and PFS (d) curves according to the level of CD8+ TILs. TILs, tumor-infiltrating lymphocytes; PFS, progression-free survival; OS, overall survival; SOX2, sex-determining region Y-box 2.

that a high number of intratumoral CD8+ TILs is significantly related with longer PFS in LS-SCLC $(p=0.014)$ in our study could be valuable. Additionally, this finding suggests that some LS-SCLCs may have a favorable tumor microenvironment characterized by preexisting CD8+ Tcells; this has been linked to the increased efficacy of immune checkpoint inhibitors [24]. In addition, Ishii et al. [25] reported that the aberrant expression of PD-L1 in tumor cells is frequently observed with LS-SCLC, compared to ES-SCLC. Taken together, these findings suggest that antitumor immunotherapy mediated through CD8+ TILs may have a crucial role in LS-SCLC.
SOX2 is implicated in the carcinogenesis in SCLC. Amplification for SOX2 was found in 27\% of SCLCs [14]. SOX2 is known to play a role in maintenance of the proliferative potential and stem cell function in cancer cells. In an in vitro study, suppression of the SOX2 gene blocked the proliferation of a SCLC cell line [14]. In a clinical study, high SOX2 expression in SCLC was observed in $55.9 \%$ of patients and was significantly associated with a poor prognosis [26]. However, in this study, we found that SOX2 expression itself was not significantly related to patient survival in SCLC ( $p=0.118)$. These differences may be due to the difference in the patient population 


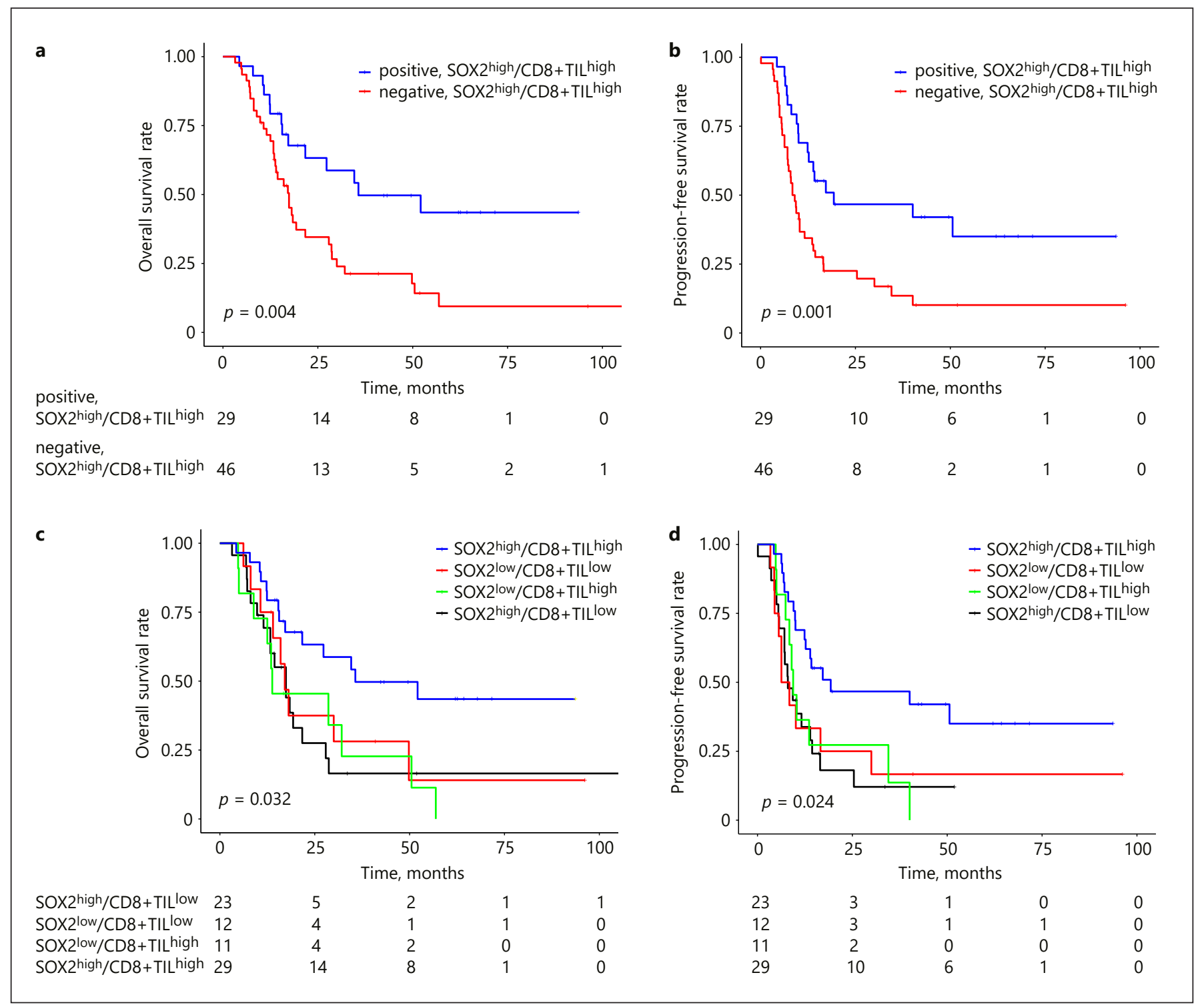

Fig. 4. Kaplan-Meier curves were plotted based on the combined analysis of SOX2 expression and CD8+ TIL levels. The patients with the combination of high SOX2 expression and levels of CD8+ TILs demonstrated longer OS (a) and PFS (b). OS (c) and PFS (d) in the 4 subgroups were plotted based on the combination of SOX2 expression and CD8+ TIL levels. TILs, tumor-infiltrating lymphocytes; PFS, progression-free survival; OS, overall survival; SOX2, sex-determining region Y-box 2.

used in this study, which was limited to LS-SCLC cases. Another reason for the different roles of SOX2 may be due to differences in the immunogenic antigens derived from tumor cells in various cancers, especially SCLC [27, 28]. Antibodies against the SOX 2 protein were observed in the serum samples of $22 \%$ of the patients with SCLC [29]. The rate of detection of SOX2 antibodies was high at $67 \%$ in patients with concomitant SCLC and LambertEaton myasthenia syndrome, a rare autoimmune disease characterized by muscle weakness [16]. Neurologic paraneoplastic syndromes, including Lambert-Eaton myasthenia syndrome, may be related to a favorable prognosis in SCLC that is induced by high CD8+ TIL numbers and levels of tumor antigens, including SOX2 [30, 31]. In our study, LS-SCLC with high SOX2 expression demonstrated a significantly increased density of CD8+ TILs ( $p=$ 0.041). According to these findings, further studies are needed to elucidate the actual role of SOX 2 through inte- 


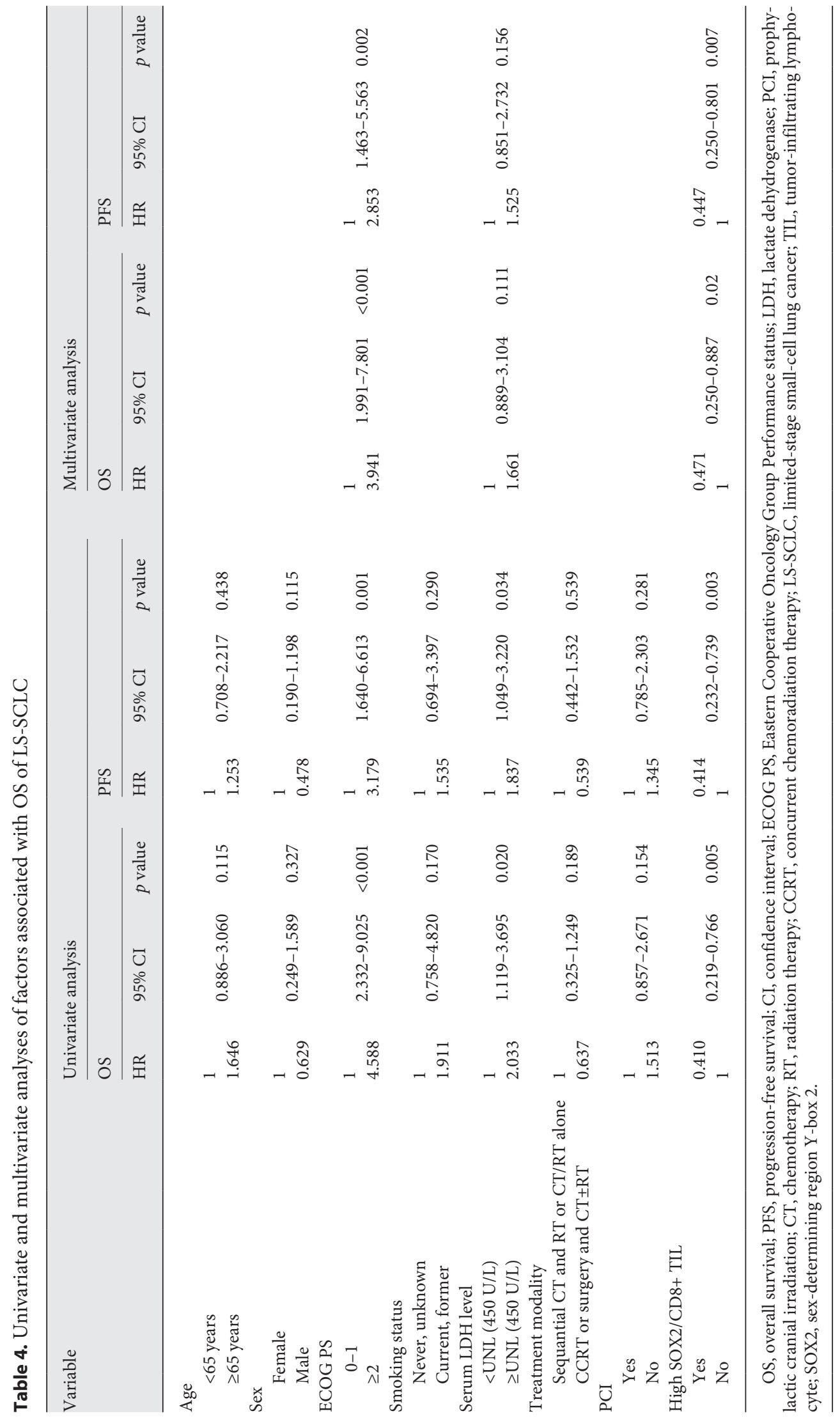


grated analysis of the oncogenic and tumor immunityassociated functions of SOX2.

We found that patients with high SOX2 expression and high CD8+ TIL numbers demonstrated a favorable prognosis, and these constitute independent prognostic factors in LS-SCLC. SOX2-specific T-lymphocytes stimulated by CD154-activated B cells and with combined immune checkpoint inhibitors have a strong tumoricidal effect on lung cancer cell lines $[32,33]$. SOX2 is also an endogenous target of T-cell immunity in non-SCLC. Interestingly, patients with SOX2-targeting T-cells in nonSCLC who were treated with an immune checkpoint blocker showed a superior response rate compared to those without SOX2-targeting T-cells [18]. Altogether, the evaluation of SOX2 expression in tumor cells and the levels of CD8+ TILs can be helpful to stratify patients with LS-SCLC according to their prognosis. In addition, further studies on the efficiency of immune checkpoint blockers on the levels of SOX2 and CD8+ TILs are needed.

Our study has a few limitations. The limitation concerns the sample size and heterogeneous treatment modality, although the majority $(86.7 \%)$ of patients received 4 cycles of etoposide, platinum, and definite local therapy (sequential chemo and radiotherapy or). The current standard of treatment for LS-SCLC is chemotherapy and concurrent thoracic radiotherapy. Chemotherapy plus radiotherapy showed a 5.4\% improvement in OS at 2 years comparing to chemotherapy alone [34]. Compared to the homogeneous platinum based systemic chemotherapy approach in ES-SCLC, LS-SCLC is treated with varied treatment modalities based on clinical characteristics such as the lung function and TNM stage. Recently, surgical resection and concurrent chemoradiotherapy are recommended for LS-SCLC with T1-2N0M0 [35]. Moreover, the study for the radiotherapy schedule, radiation dose, and the candidate for prophylactic cranial irradiation has been continuously conducted to improve the prognosis of patients with LS-SCLC $[36,37]$. Therefore, further studies using well-defined patient groups based on the patients' treatment methods are needed and can be helpful to address an additional role of SOX2 and CD8+ TIL in LS-SCLC.

\section{Conclusion}

Our study showed the prognostic role of SOX2 in association with CD8+ TILs in LS-SCLC. The SOX2 expression itself is not significantly related to patient survival.
However, concomitantly high SOX2 and CD8+ TIL levels are significantly associated with longer OS and PFS and represent independent favorable prognostic factors in LS-SCLC. Studies on the interaction between SOX2 and tumor immunity in SCLC will provide a better understanding of tumor immunology and improve the efficacy of immunotherapy in SCLC.

\section{Statement of Ethics}

All procedures followed were in accordance with the ethical standards of the Responsible Committee on human experimentation and with the Helsinki Declaration of 1964 and later versions. Our study was approved by the Seoul St. Mary's Hospital's Institutional Review Board for (protocol KC18SES10552), where written informed consent or a substitute for it was obtained from all patients included in the study.

\section{Conflict of Interest Statement}

The authors declare that they have no conflicts of interest.

\section{Funding Sources}

This study was supported by Research Fund of Seoul St.Mary's Hospital and Medical collge, The Catholic University of Korea..

\section{Author Contributions}

J.L.: conception and design, collection and assembly of data, data analysis and interpretation, writing, and approval of the final report. Y.Y.J., J.H.L., and H.-W.H.: collection and assembly of data, and approval of the final report. M.H.: conception and design, data analysis and interpretation, editing, and approval of the final report. S.-H.H. and S.A.H: conception and design, collection and assembly of data, data analysis, and interpretation, writing and editing, approval of the final report, and responsibility for overall content.

\section{References}

1 Sabari JK, Lok BH, Laird JH, Poirier JT, Rudin CM. Unravelling the biology of SCLC: implications for therapy. Nat Rev Clin Oncol. 2017 Sep;14(9):549-61.

2 Pignon JP, Arriagada R, Ihde DC, Johnson DH, Perry MC, Souhami RL, et al. A metaanalysis of thoracic radiotherapy for smallcell lung cancer. N Engl J Med. 1992 Dec 3; 327(23):1618-24.

3 Turrisi AT 3rd, Kim K, Blum R, Sause WT, Livingston RB, Komaki R, et al. Twice-daily compared with once-daily thoracic radiotherapy in limited small-cell lung cancer treated concurrently with cisplatin and etoposide. N Engl J Med. 1999 Jan 28;340(4):265-71. 
4 Nicholson AG, Chansky K, Crowley J, Beyruti R, Kubota K, Turrisi A, et al. The international association for the study of lung cancer lung cancer staging project: proposals for the revision of the clinical and pathologic staging of small cell lung cancer in the forthcoming eighth edition of the TNM classification for lung cancer. J Thorac Oncol. 2016;11(3):30011.

5 Vallieres E, Shepherd FA, Crowley J, Van Houtte P, Postmus PE, Carney D, et al. The IASLC lung cancer staging project: proposals regarding the relevance of TNM in the pathologic staging of small cell lung cancer in the forthcoming (seventh) edition of the TNM classification for lung cancer. J Thorac Oncol. 2009;4(9):1049-59.

6 Horn L, Mansfield AS, Szczęsna A, Havel L, Krzakowski M, Hochmair MJ, et al. First-line atezolizumab plus chemotherapy in extensive-stage small-cell lung cancer. N Engl J Med. 2018;379(23):2220-9.

7 Paz-Ares L, Dvorkin M, Chen Y, Reinmuth N, Hotta K, Trukhin D, et al. Durvalumab plus platinum-etoposide versus platinum-etoposide in first-line treatment of extensive-stage small-cell lung cancer (CASPIAN): a randomised, controlled, open-label, phase 3 trial. Lancet. 2019;394(10212):1929-39.

8 Higgins KA, Gorgens S, Sudmeier LJ, FaivreFinn C. Recent developments in limited stage small cell lung cancer. Transl Lung Cancer Res. 2019;8(Suppl 2):S147.

9 McGranahan N, Furness AJ, Rosenthal R, Ramskov S, Lyngaa R, Saini SK, et al. Clonal neoantigens elicit $\mathrm{T}$ cell immunoreactivity and sensitivity to immune checkpoint blockade. Science. 2016 Mar 25;351(6280):1463-9.

10 Donnem T, Hald SM, Paulsen EE, Richardsen E, Al-Saad S, Kilvaer TK, et al. Stromal CD8+ T-cell density: a promising supplement to TNM staging in non-small cell lung cancer. Clin Cancer Res. 2015 Jun 1;21(11):2635-43.

11 Farhood B, Najafi M, Mortezaee K. CD8(+) cytotoxic T lymphocytes in cancer immunotherapy: a review. J Cell Physiol. 2019 Jun; 234(6):8509-21.

12 Matsushita H, Vesely MD, Koboldt DC, Rickert CG, Uppaluri R, Magrini VJ, et al. Cancer exome analysis reveals a T-cell-dependent mechanism of cancer immunoediting. $\mathrm{Na}$ ture. 2012 Feb 8;482(7385):400-4.

13 Zhang S, Cui W. Sox2, a key factor in the regulation of pluripotency and neural differentiation. World J Stem Cells. 2014 Jul 26;6(3): 305-11.

14 Rudin CM, Durinck S, Stawiski EW, Poirier JT, Modrusan Z, Shames DS, et al. Comprehensive genomic analysis identifies SOX2 as a frequently amplified gene in small-cell lung cancer. Nat Genet. 2012 Oct;44(10):1111-6.
15 Vural B, Chen LC, Saip P, Chen YT, Ustuner $\mathrm{Z}$, Gonen $\mathrm{M}$, et al. Frequency of SOX Group B (SOX1, 2, 3) and ZIC2 antibodies in Turkish patients with small cell lung carcinoma and their correlation with clinical parameters. Cancer. 2005 Jun 15;103(12):2575-83.

16 Titulaer MJ, Klooster R, Potman M, Sabater L, Graus F, Hegeman IM, et al. SOX antibodies in small-cell lung cancer and Lambert-Eaton myasthenic syndrome: frequency and relation with survival. J Clin Oncol. 2009 Sep 10; 27(26):4260-7.

17 Sun Y, Zhang R, Wang M, Zhang Y, Qi J, Li J. SOX2 autoantibodies as noninvasive serum biomarker for breast carcinoma. Cancer Epidemiol Biomarkers Prev. 2012 Nov;21(11): 2043-7.

18 Dhodapkar KM, Gettinger SN, Das R, Zebroski H, Dhodapkar MV. SOX2-specific adaptive immunity and response to immunotherapy in non-small cell lung cancer. Oncoimmunology. 2013 Jul 1;2(7):e25205.

19 Hong SA, Hwang HW, Kim MK, Lee TJ, Yim $\mathrm{K}$, Won HS, et al. High endothelial venule with concomitant high CD8+ tumor-infiltrating lymphocytes is associated with a favorable prognosis in resected gastric cancer. J Clin Med. 2020;9(8):2628.

20 Kalemkerian GP, Schneider BJ. Advances in small cell lung cancer. Hematol Oncol Clin North Am. 2017 Feb;31(1):143-56.

21 Perez BA, Kim S, Wang M, Karimi AM, Powell C, Li J, et al. A prospective single arm phase I/II study: ipilimumab and nivolumab with thoracic radiotherapy after platinum chemotherapy in extensive-stage small cell lung cancer. Int J Radiat Oncol Biol Phys. 2020; 109(2)-35.

22 Chen P, Zhang L, Zhang W, Sun C, Wu C, He $\mathrm{Y}$, et al. Galectin-9-based immune risk score model helps to predict relapse in stage I-III small cell lung cancer. J Immunother Cancer. 2020 Oct; $8(2): \mathrm{e} 001391$

23 Sun C, Zhang L, Zhang W, Liu Y, Chen B, Zhao S, et al. Expression of PD-1 and PD-L1 on tumor-infiltrating lymphocytes predicts prognosis in patients with small-cell lung cancer. Onco Targets Ther. 2020;13:6475-83.

24 Thomas A, Vilimas R, Trindade C, Erwin-Cohen R, Roper N, Xi L, et al. Durvalumab in combination with olaparib in patients with relapsed SCLC: results from a phase II study. J Thorac Oncol. 2019 Aug;14(8):1447-57.

25 Ishii H, Azuma K, Kawahara A, Yamada K, Imamura Y, Tokito T, et al. Significance of programmed cell death-ligand 1 expression and its association with survival in patients with small cell lung cancer. J Thorac Oncol. 2015 Mar; 10(3):426-30.
26 Yang F, Gao Y, Geng J, Qu D, Han Q, Qi J, et al. Elevated expression of SOX2 and FGFR1 in correlation with poor prognosis in patients with small cell lung cancer. Int J Clin Exp Pathol. 2013;6(12):2846.

27 Maddison P, Thorpe A, Silcocks P, Robertson JF, Chapman CJ. Autoimmunity to SOX2, clinical phenotype and survival in patients with small-cell lung cancer. Lung Cancer. 2010 Dec;70(3):335-9.

28 Atakan S, Bayiz H, Sak S, Poyraz A, Vural B, Yildirim AS, et al. Autologous anti-SOX2 antibody responses reflect intensity but not frequency of antigen expression in small cell lung cancer. BMC Clin Pathol. 2014;14(1):24.

29 Güre AO, Stockert E, Scanlan MJ, Keresztes RS, Jäger D, Altorki NK, et al. Serological identification of embryonic neural proteins as highly immunogenic tumor antigens in small cell lung cancer. Proc Natl Acad Sci USA. 2000;97(8):4198-203.

30 Albert ML, Darnell RB. Paraneoplastic neurological degenerations: keys to tumour immunity. Nat Rev Cancer. 2004 Jan;4(1):36-44.

31 Iams WT, Shiuan E, Meador CB, Roth M, Bordeaux J, Vaupel C, et al. Improved prognosis and increased tumor-infiltrating lymphocytes in patients who have SCLC with neurologic paraneoplastic syndromes. J Thorac Oncol. 2019 Nov; 14(11):1970-81.

32 Zhang X, Zhang Y, Xu J, Wang H, Zheng X, Lou Y, et al. Antigen presentation of the Oct4 and Sox 2 peptides by CD154-activated B lymphocytes enhances the killing effect of cytotoxic T lymphocytes on tumor stem-like cells derived from cisplatin-resistant lung cancer cells. J Cancer. 2018;9(2):367-74.

33 Zhang X, Hu F, Li C, Zheng X, Zhang B, Wang $\mathrm{H}$, et al. OCT4\&SOX2-specific cytotoxic T lymphocytes plus programmed cell death protein 1 inhibitor presented with synergistic effect on killing lung cancer stem-like cells in vitro and treating drug-resistant lung cancer mice in vivo. J Cell Physiol. 2019 May;234(5): 6758-68

34 Warde P, Payne D. Does thoracic irradiation improve survival and local control in limitedstage small-cell carcinoma of the lung? A meta-analysis. J Clin Oncol. 1992 Jun;10(6):8905.

35 Zhong L, Suo J, Wang Y, Han J, Zhou H, Wei $\mathrm{H}$, et al. Prognosis of limited-stage small cell lung cancer with comprehensive treatment including radical resection. World J Surg Oncol. 2020;18(1):27.

36 Stinchcombe TE. Current treatments for surgically resectable, limited-stage, and extensive-stage small cell lung cancer. Oncologist. 2017 Dec;22(12):1510-7.

37 Daly ME, Ismaila N, Decker RH, Higgins K, Owen D, Saxena A, et al. Radiation therapy for small-cell lung cancer: ASCO guideline endorsement of an ASTRO guideline. J Clin Oncol. 2021;39(8):931-9. 